\title{
Single-cell whole-genome sequencing identifies human papillomavirus integration in cervical tumour cells prior to and following radiotherapy
}

\author{
DONG YANG ${ }^{1}$, WEIYUAN ZHANG ${ }^{1}$, YANG LIU ${ }^{1}$, JUNQING LIANG ${ }^{2}$, TONGQING ZHANG ${ }^{1}$, \\ YUNBO BAI ${ }^{1}$, WENJING HAO ${ }^{1}$, KEXIN MA $^{1}$, DANNI LU ${ }^{1}$ and JING CHEN ${ }^{3}$ \\ ${ }^{1}$ Department of Gynaecology, Beijing Obstetrics and Gynaecology Hospital, Capital Medical University, \\ Beijing 100026; ${ }^{2}$ Department of Gynaecology, Peking University People's Hospital, Beijing 100044; \\ ${ }^{3}$ Department of Gynaecology, Beijing Youan Hospital, Capital Medical University, Beijing 100069, P.R. China
}

Received August 30, 2016; Accepted October 13, 2017

DOI: $10.3892 / \mathrm{ol} .2018 .8567$

\begin{abstract}
Single-cell sequencing technology is a promising systematic and comprehensive approach to delineate clonal associations between cells. The present study collected 13 and 12 cervical cells from fresh tumour tissue prior to and following radiotherapy, respectively, from a 46-year-old female patient with exogenous-type cervical carcinoma. Next, single-cell whole-genome sequencing analysis was performed on each cell. Examination revealed that normal cells could be clearly distinguished from tumour cells among the 25 cells. Tumour cells prior to and following radiotherapy almost represented two independent clones, with the main subpopulation prior to radiotherapy being killed and the minor subpopulation prior to radiotherapy becoming the main subpopulation following radiotherapy. A human papillomavirus (HPV) integration site was detected in POU class 5 homeobox 1B (POU5F1B) in tumour cells following radiotherapy, which has been reported to be a frequent HPV integration site in cervical carcinoma. These results indicate that tumour cells with HPV integration in POU $F 1 B$ survive radiotherapy, and that tumour cells prior to and following radiotherapy exhibit distinct characteristics.
\end{abstract}

\section{Introduction}

Cervical cancer is one of the most common malignant tumours in the female reproductive system, with $\sim 500,000$ new cases each year worldwide (1), accounting for $5 \%$ of all new cancer

Correspondence to: Dr Weiyuan Zhang, Department of Gynaecology, Beijing Obstetrics and Gynaecology Hospital, Capital Medical University, 251 Yaojiayuan Road, Beijing 100026, P.R. China

E-mail: zhangwy9921@hotmail.com

Key words: single-cell sequencing, cervical cancer, radiotherapy, somatic mutation, human papillomavirus cases. Overall, $78 \%$ of cases are reported in developing countries (2).

Radiotherapy is one of the most well-used tumour treatments (3). As reported by the World Health Organization in $1992,60-70 \%$ of patients with malignant tumours undergo radiotherapy (4). Approximately $45 \%$ of all tumours can be cured using a variety of treatments, $22 \%$ of which can be cured by surgery, $18 \%$ by radiotherapy and $5 \%$ by chemotherapy (5). Thus, radiotherapy is valuable in tumour treatment. With the widespread use of precise radiotherapies, including conformal radiotherapy, intensity-modulated radiotherapy,image-guarded radiation therapy, and the application of protons and heavy ions in the clinic, the role of radiotherapy has become increasingly important in tumour therapy (6). However, in clinical practice, the majority of radiation practitioners have found that not every patient with a tumour responds to the treatment $(7,8)$. Certain patients only exhibit good responses at the beginning of treatment. Radiation can control the tumour, provide detailed imaging scans and clinical efficacy $(7,8)$. However, local recurrence or distant metastasis can occur shortly after treatment (9). A second course of irradiation does not usually produce good results (9). To solve this problem, the changes that occur in tumour genomes following radiotherapy require further understanding.

In traditional research, individual cells of the same phenotype have been commonly viewed as identical functional units of a tissue (10). Analyses by conventional detection methods are always based on the overall average reaction of cells (10). Sequencing of DNA or RNA from single cells indicates that heterogeneous cells enable the system-level functions of a tissue (11). Single-cell sequencing is a type of high-throughput sequencing technology at the single-cell level. Compared with conventional throughput sequencing, it is a powerful approach for studying the genetic heterogeneity of individual cells with the same phenotype (11).

Human papillomavirus (HPV) infection is a major cause of cervical cancer $(12,13)$. In China, HPV infection is also prevalent (14-16). Recently, using high throughput sequencing, $\mathrm{Hu}$ et al (17) reported frequent HPV integration sites in genes such as POU class 5 homeobox 1B (POU5F1B) $(9.7 \%)$ and 
fragile histidine triad $(8.7 \%)$. However, to the best of our knowledge, there are no reports concerning HPV infection prior to and following radiotherapy.

Navin et al (18) applied single-nucleus sequencing to investigate the tumour population structure and evolution in two human breast cancer cases. Each analysis of 100 single cells from the two cases revealed three distinct clonal subpopulations in one heterogeneous tumour, whereas another tumour consisted of a group of genetically identical cells (12). This data indicated that tumours grow by punctuated clonal expansions, with few persistent intermediates. Xu et al (19) performed single-cell exome sequencing of renal cell carcinoma, revealing that the tumour did not contain any significant clonal subpopulations, and demonstrating that mutations occurred at different frequencies and different mutation spectrums. The study demonstrated that renal cell carcinoma maybe more heterogeneous than was believed, which would require the development of more effective cellular targeted therapies (13). This approach is also conducive for researching the mechanism of tumour development and metastasis. Felthaus et al (20) analysed oral squamous cell carcinoma cell lines and revealed that the resistance of this cancer to conventional chemotherapy or radiotherapy may be caused by cancer stem cells.

In view of the power of single-cell sequencing technology, the present study analysed genomic alterations, particularly in terms of HPV infection, prior to and following radiotherapy. Furthermore, using this technology, the effect of radiotherapy could be assessed in patients with cervical cancer and guide subsequent treatment in the future.

\section{Materials and methods}

Sample collection and preparation of cell suspensions. Fresh tumour and blood samples were obtained from a 46-year-old female patient with the exogenous type of cervical carcinogenesis at Beijing Obstetrics and Gynaecology Hospital (Beijing, China) in April 2015. The diagnosis of cervical carcinogenesis has been described in detail previously (17). The pathological type of cervical cancer was squamous cell carcinoma and the tumour was classified as stage IIA2, according to the 2009 International Federation of Gynaecology and Obstetrics staging system (21). The size of the primary tumour was $5 \mathrm{~cm}$. The HPV type was detected as HPV 16 using flow-through hybridization. The level of squamous cell carcinoma antigen was $4.74 \mu \mathrm{g} / \mathrm{l}$. The patient received $10 \mathrm{~Gy}$ in 5 fractions of 2 Gy, following which the tumor tissue was excised and 12 cells were isolated for gene sequencing. Then, the patient continued to receive $36 \mathrm{~Gy}$ in 18 fractions of $2 \mathrm{~Gy}$ (10 Gy). Following radiation therapy, the level of squamous cell carcinoma antigen was $4.62 \mu \mathrm{g} / \mathrm{l}$. No improvements were noted in the patient's condition. Tumour tissues were obtained prior to and following radiotherapy. The tumour tissues were pathologically confirmed as malignant cervical carcinogenesis with $>90 \%$ tumour cells. The present study was performed with the approval of the Beijing Obstetrics and Gynaecology Hospital. Signed written consent was obtained from the patient prior to recruitment to the study.

Collection of single cells and preparation of cell lysates. Single cells from the tumour samples were prepared as described previously (19) A manually controlled pipetting system was used to isolate single cells under an inverted light microscope (Nikon Instruments Co., Ltd.). Each cell was transferred into a precooled polymerase chain reaction (PCR) tube containing a cell lysis solution (Qiagen $\mathrm{GmbH}$, Hilden, Germany) (The samples were incubated in a thermocycler for $10 \mathrm{~min}$ at $65^{\circ} \mathrm{C}$. A physiological saline blank was included as a negative control. Every step during the experiments was performed strictly according to the aforementioned protocol. With sufficient dispersion and cascade-dilution of the cells, single cells were randomly isolated from tumour tissues into PCR-ready tubes using an inverted microscope and a mouth-controlled, fine hand-drawn microcapillary pipetting system made in-house. Single-cell isolation was visually confirmed by microscopy and documented as micrographs. The cells were washed three times using the elution buffer (Qiagen $\mathrm{GmbH}$ ).

Multiple displacement amplification (MDA). Whole-genome amplification (WGA) was performed using a REPLI-g Mini kit (Qiagen $\mathrm{GmbH}$ ) according to the manufacturer's protocol. All samples were amplified by MDA, according to the aforementioned protocol. A total reaction volume of $50 \mu \mathrm{l}$ was used at $30^{\circ} \mathrm{C}$ for $16 \mathrm{~h}$ and then terminated at $65^{\circ} \mathrm{C}$ for $10 \mathrm{~min}$. Amplified DNA products were then stored at $-20^{\circ} \mathrm{C}$.

Whole-genome sequencing (WGS). Paired-end library preparation was conducted using Illumina protocols (22). Genomic DNA (400 ng) was fragmented to an insert size of $~ 400 \mathrm{bp}$ with a Covaris device (M220 Focused-ultrasonicator; Covaris, Inc., Woburn, MA, USA), and size selection was performed using $2 \%$ agarose gel excision. Deep sequencing was performed using Illumina X10 instruments (Illumina, Inc., San Diego, CA, USA). Each sample achieved $~ 38$ times genomic coverage.

Somatic mutation detection. Following the removal of adapters and low-quality reads, all sequencing reads were mapped to the human genome (hg19 build) (23) using Burrows-Wheeler Aligner (v0.5.9) (24) with default parameters. The sequence alignment map output was converted to a sorted binary alignment map file using SAM tools (v1.3) (25). Picard (v1.70) (Broad Institute, Cambridge, MA, USA) was used to remove PCR duplicates. Somatic single nucleotide variants (SNVs) were detected by VarScan (v2.3.9) (26). A candidate somatic mutation was called if the following criteria were met: i) The somatic P-values of the variants were $<0.05$; ii) mutant allele frequencies in tumour cells were $>15 \%$; iii) mutant allele frequencies in the normal control were $<0.5 \%$; iv) reads with a mutant allele were $>4$; v) the forward reference count (i.e., the number of forward reads that match the reference base at the locus), the reverse reference count (i.e., the number of reverse reads that match the reference base at the locus), the forward non-reference count (i.e., the number of forward reads that do not match the reference base at the locus) and the reverse non-reference count (i.e., the number of reverse reads that do not match the reference base at the locus) in the tumour must be $\geq 1$; and vi) only mutations detected in four samples were retained. To eliminate common germline variants, SNVs observed in dbSNP137 or the 1,000 Genomes Project, March 2012 data release project were excluded (27). Annotation was performed using snpEff (v4.2) (28). GR Ch37.75 was used 
for transcript identification and to determine amino acid changes. All putative somatic mutations in coding regions were validated visually. The mean number and the standard deviation of somatic mutations were calculated. When it was found that the number of somatic mutations in one sample was less than the total of the mean number minus the standard deviation, the sample with the fewest number of somatic mutations was removed and this sample was considered as the normal cells. In this way, when the sample with 25 somatic mutations was removed, the number of all samples remained was greater than the total of the mean number minus the standard deviation.

Mapping and analysis of HPV integration sites. The 400-bp paired-end fragment libraries (150 bp read length) were mapped to the human reference genome (hg19) and HPV genome (HPV6: FR751337.1, HPV82: AF293961.1, HPV69: AB027020.1, HPV68: FR7 51039.1, HPV66: EF177191.1, HPV59: E U918767.1, HPV58: HQ537777.1, HPV56: EF177181.1, HPV52: HQ537751.1, HPV45: EF202167.1, HPV39: M62849.1, HPV35: HQ537730.1, HPV33: HQ537688.1, HPV31: HQ537687.1, HPV18: AY262282.1, HPV16: NC_001526.2, and HPV11: HE574705.1; www.ncbi.nlm.nih.gov). If a paired-end read was uniquely mapped to hg19 at one end and to HPV at the other end, integration was reported. All mapping locations were subjected to a filtering process to remove possible PCR duplicates. Specifically, if there were two or more paired reads that mapped to near-identical locations $( \pm 2 \mathrm{bp})$, only one of the reads was considered. The fusion point was determined by analysing cases in which one region of the read aligned to HPV and the other aligned to hg19. These locations were crosschecked with the clusters of paired-end reads for consistency. Furthermore, to determine the exact fusion point between hg19 and HPV, all hg19-HPV mapped reads were extracted, and aligned each read entirely on hg19 and HBV using Blat (http://genome.ucsc.edu/cgi-bin/hgBlat, -min -Score 25, -minIdentity 85).

Statistical analysis. Paired Student's t-test was used to compare the number of somatic mutations in samples prior to and following radiotherapy. The Wilcoxon rank-sum test was used to compare the HPV integration events between tumour and normal cells or tumor cells prior to and following radiotherapy. $\mathrm{P}<0.05$ was considered to indicate a statistically significant difference. All tests were performed using R software (v2.14; https://www.R-project.org).

\section{Results}

High-throughput isolation and amplification of single cells from fresh tumour tissues. Fresh tumour tissues prior to and following radiotherapy were obtained from a 46-year-old woman with cervical carcinoma classified as stage IIA2. Blood was also collected from this patient, which was used as a matched normal control. To obtain detailed cellular genetic information on this tumour, single cell sequencing in individual cells from the tumour samples was performed as described previously (19). WGA was performed based on MDA of the DNA from each single cell of the tumour tissues. In total, 13 cells were obtained from tumour tissues prior to radiotherapy (SZ1512000007-SZ1512000019), and 12 cells were obtained from tumour tissues following radiotherapy (SZ1512000020-SZ1512000031). These 25 single tumour cells met the previously described criteria (19) and were selected for subsequent analysis. Massively parallel single-cell WGS was performed on these samples using paired-end 150-bp reads. The blood sample also underwent conventional WGS. A mean of $114 \mathrm{~Gb}$ of high-quality mappable WGS data were aligned to the human reference genome, with 38.0-fold coverage in the tumour prior to radiotherapy, 37.8-fold coverage in the tumour following radiotherapy and 38.6-fold coverage in the blood (Fig. 1).

To detect somatic mutations in each cell, Varscan (v2.3.9) was used to assess tumour-normal pairs, with each tumour cell as tumour and the blood sample as normal. To avoid variation-calling biases in single cell sequencing, only mutations that were detected in at least four samples were retained. Following the removal of somatic mutations that occurred at dbSNP135 and in the 1,000 Genomes Project March 2012 data release project, 149 somatic protein-altering mutations were identified in the 25 samples. Samples harboured mean of 47 somatic mutations. Analysis of all somatic mutations showed a preference for $\mathrm{C}>\mathrm{T} / \mathrm{G}>\mathrm{A}$, the apolipoprotein $\mathrm{B}$ mRNA editing enzyme catalytic subunit 3Bmutation signature (29), and was similar to the previously reported mutation mechanism pattern observed in cervical cancer (30). A total of 7 samples with $<25$ mutations were recognised as normal cells owing to the small amount of variance between sample and adjacent tissues (3 samples were obtained from tumours prior to radiotherapy and 4 samples were from tumours following radiotherapy). This is an explainable phenomenon, as tumour tissues are mixed tissues, which may be mixed with normal cells. In pre-radiotherapy samples, mean of 51.5 mutations were detected, whereas 70.5 mutations were detected in samples following radiotherapy. Significantly more somatic mutations were identified in samples following radiotherapy $(\mathrm{P}=0.030$; single-end Student's t-test; Fig. 2), suggesting that radiotherapy may cause genetic instability (31).

Principal component analysis and hierarchical cluster tree analysis. To investigate the intercellular heterogeneity structure in tumours prior to and following radiotherapy, population genetic analyses was applied to the comprehensive 25 WGS data sets. A total of 149 non-synonymous mutations were subjected to principal component analysis (PCA) to characterise the genetic heterogeneity of this tumour. It was found that two first principal components ( $\mathrm{PC1}$ and $\mathrm{PC} 2$ ), which represented $26 \%$ of the whole inertia (Table I), clearly divided isolated single cells into two subsets: Normal-cell and tumour-cell subsets (Fig. 3A). The normal-cell subset consisted of the 7 samples recognised as normal cells with $<25$ somatic mutations. The tumour-cell subsets were also clustered into two subgroups that could almost distinguish samples prior to and following radiotherapy (Fig. 3A). Next, hierarchical cluster tree analysis of the 18 tumour cells was performed to infer their population substructure. Samples from the tumour prior to radiotherapy were clustered together, as were the samples from the tumour following radiotherapy (Fig. 3B). This observation indicated the clear genetic variance between tumour cells prior to and following radiotherapy. 


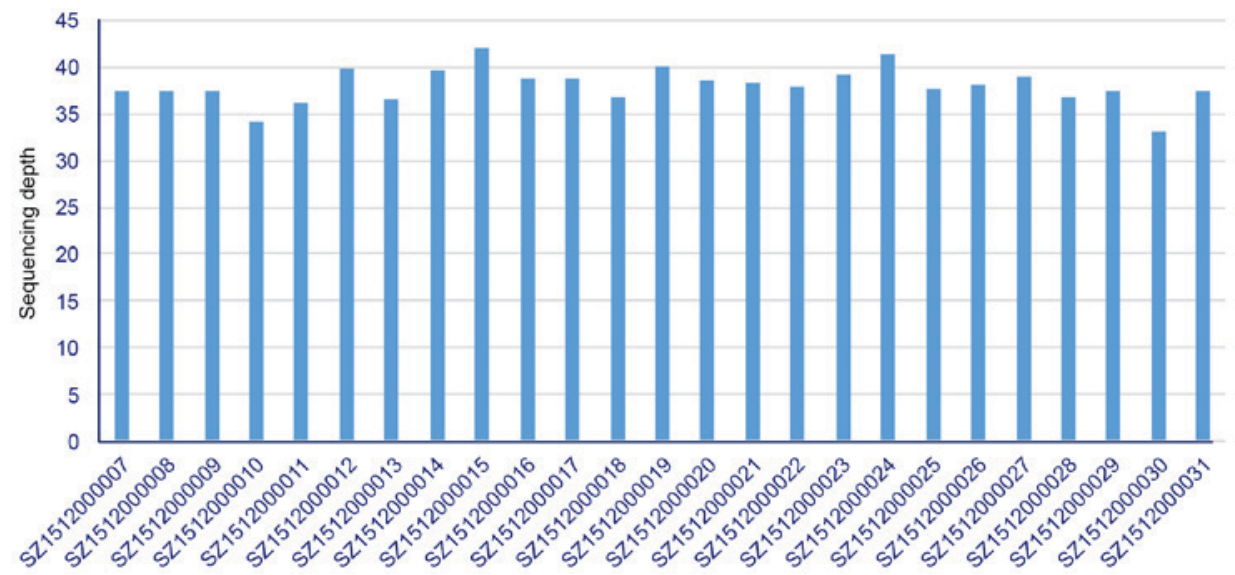

Figure 1. Sequencing depth of single cell sequencing. Distribution of the sequencing depth of the 25 samples. All samples had $>30$ times genome coverage, which was adequate for somatic mutation calling and population genetic analysis.

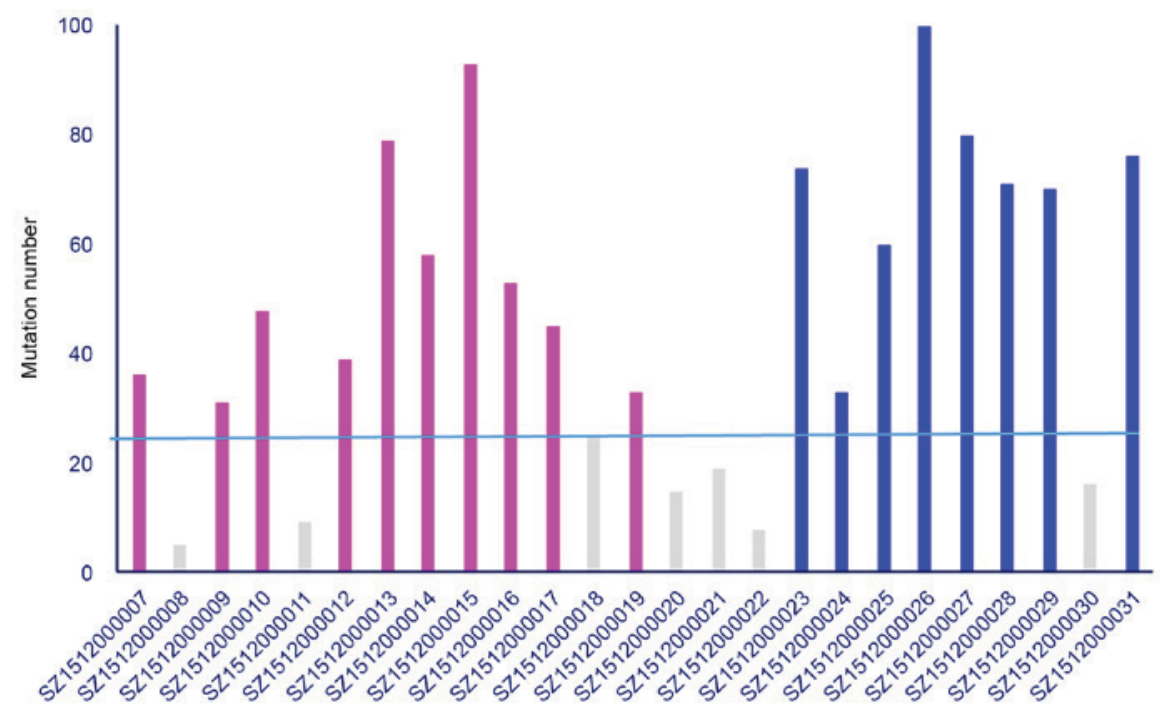

Figure 2. Identification of somatic mutations in the 25 samples. The numbers of somatic mutations identified in the 25 samples are shown. There were 7 samples considered as non-tumour cells, shown in grey. The 10 tumour samples prior to radiotherapy are shown in pink, and the 8 tumour samples following radiotherapy are shown in blue.

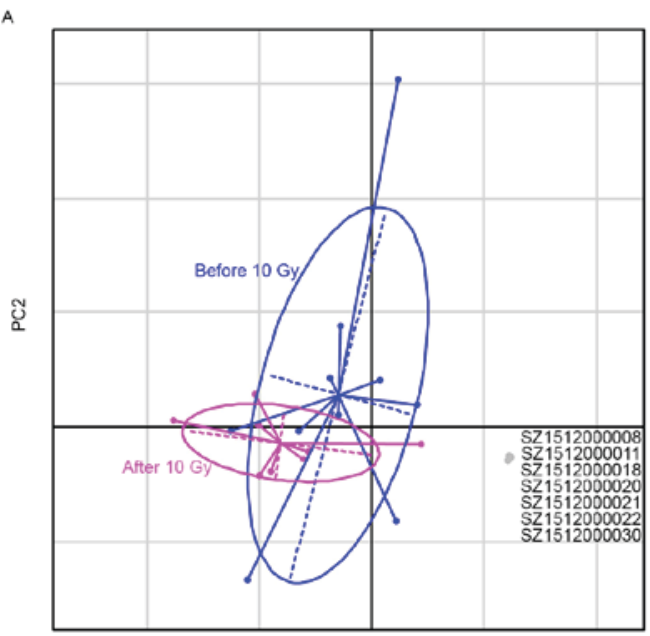

PC1
B

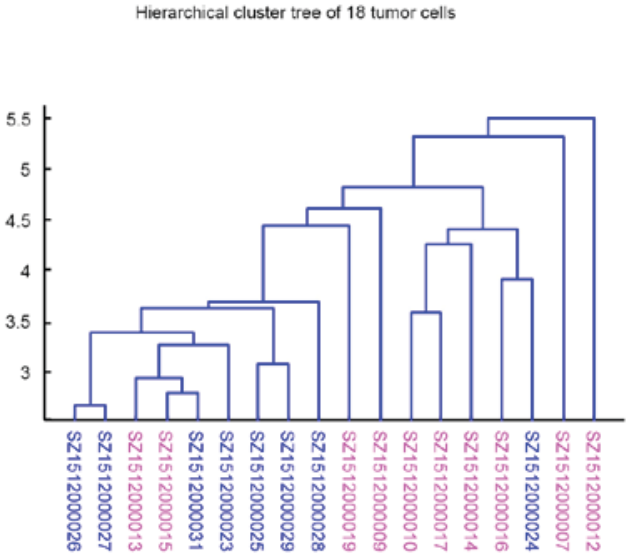

Figure 3. Population genetic analyses of somatic mutations. (A) Principle component analysis of non-synonymous mutations in tumour cells and adjacent tissues. The value of allele frequency for each non-synonymous mutated site was used. There were 7 samples considered to be non-tumour cells, shown in grey. Tumour samples following radiotherapy are shown in pink, and tumour samples prior to radiotherapy are shown in blue. (B) The 18 tumour samples were used to compute the phylogenetic association of each cell pair to build the hierarchical tree. PC, principal component. 
Table I. Percentage of each PC as a proportion of the whole inertia.

\begin{tabular}{lc}
\hline PC & Percentage \\
\hline PC1 & 16.40 \\
PC2 & 9.30 \\
PC3 & 8.50 \\
PC4 & 7.60 \\
PC5 & 7.20 \\
\hline
\end{tabular}

PC, principal component.

Notably, 8 tumour cells prior to radiotherapy and 1 tumour cell following radiotherapy were clustered together, while only 2 tumour cells prior to radiotherapy and 7 tumour cells following radiotherapy were clustered together (Fig. 3B). This result indicated that at least 2 subpopulations existed in the tumour prior to radiotherapy (the subpopulation of 8 tumour cells prior to radiotherapy were defined as subpopulation 1 , and the subpopulation of only 2 tumour cells prior to radiotherapy were defined as subpopulation 2). Subpopulation 1 was the main population in the tumour prior to radiotherapy. Following radiotherapy, the majority of the cells in subpopulation 1 were killed, and the majority of the cells in subpopulation 2 had survived. Thus, following radiotherapy, although the 2 subpopulations existed, subpopulation 2 became the main population.

$H P V$ integration analyses. To identify driver mutations in the tumour genome, which may be responsible for the radiotherapy resistance, HPV integration was detected. The patient was infected with HPV16. A total of 17 HPV-integration genes were identified in the 25 cells from the cervical carcinoma (Table II). Among these genes, an integration site in alkylglycerone phosphate synthase (AGPS) was supported by the highest number of paired-reads (512 reads). Notably, the number of sequencing reads of HPV-integration events in the 7 samples regarded as normal cells were significantly less than that in other samples $\left(\mathrm{P}=1.9 \times 10^{-4}\right.$; Wilcoxon rank-sum test; 5.0 vs. 27.7 ; Fig. 4 ), further supporting that these 7 samples were normal cells. This result was consistent with the results of a previous study (32). No significant difference was found in the number of sequencing reads of HPV integration events between samples from the tumour prior to and following radiotherapy $(\mathrm{P}=1.0$, Wilcoxon rank-sum test).

A total of 4 and 1 sequencing reads of HPV integration events were identified in POU5F1B of two different samples from the tumour following radiotherapy, respectively; $P O U 5 F 1 B$ has been reported as a frequently integrated gene $(17,33)$. The supporting reads to the human and HPV genome were remapped using Blat (UCSC Genome Browser), and it was found that there were no artefacts. However, no evidence of HPV integration events was found in POU5F1B in samples from the tumour prior to radiotherapy.

The most likely explanation for this phenomenon is that the tumour tissue prior to radiotherapy was of polyclonal origin. The tumour cells with HPV integrations in $P O U 5 F 1 B$

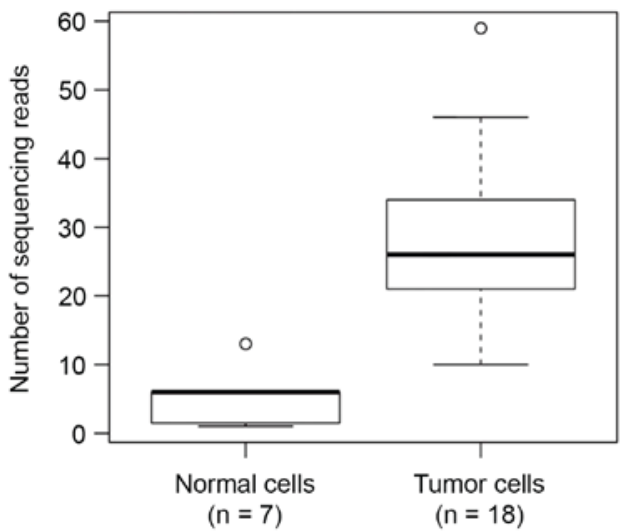

Figure 4. HPV integration in cervical cancer cells and normal cells. The number of chimeric reads (a paired-end read uniquely mapped with one end to hg19 and the other to HPV) in normal and tumour cells. The Wilcoxon rank-sum test was used to compare the difference between two groups $(\mathrm{P}=1.9 \times 10-4)$. HPV, human papillomavirus.

represent only a small proportion of these clones. Thus, the integration events in POU5F1B could not be detected by WGS. Following radiotherapy, tumour cells with HPV integrations in POU5F1B had survived, whereas the majority of other cells were killed by the radiotherapy. Consequently, the proportion of tumour cells with HPV integrations in POU 5F1B increased. Thus, this integration could be detected by WGS. The genetic mechanism under radiotherapy resistance is complex. The HPV integration site in POU 5F1B was only one of the genetic mechanisms that caused radiotherapy resistance. The development of radiotherapy resistance may be due to somatic mutations, copy number variants or structure variants. Thus, further study is required.

AGPS was the most common integration gene in all the cells. The number of AGPS integrations in tumour cells was significantly higher than that in normal cells $(\mathrm{P}=0.00040$; Wilcoxon rank-sum test; 26.7 vs. 4.6). However, there was no significant difference in the number of AGPS integrations between cells prior to and following radiotherapy $(\mathrm{P}=0.89)$. In fact, no evidence that AGPS was associated with radiotherapy resistance was found in previous reports either.

Taken together, these results indicated that tumour cells prior to and following radiotherapy exhibit distinct genetic characteristics, and tumour cells with HPV integrations in $P O U 5 F 1 B$ are more likely to survive following radiotherapy.

\section{Discussion}

The present study performed single-cell sequencing on 25 cells from cervical tumour samples prior to and following radiotherapy. WGS was also performed as a normal control for the 25 tumour cells. To the best of our knowledge, this is the first detailed genetic landscape of a tumour prior to and following radiotherapy at the single-cell level.

Population analysis of identified somatic mutations allowed for tumour cells to be distinguished from normal cells. The number of somatic mutations in cells from tumours following radiotherapy was significantly higher than that in cells from tumours prior to radiotherapy. Through PCA and hierarchical cluster tree analysis, it was found that cells from the tumour 


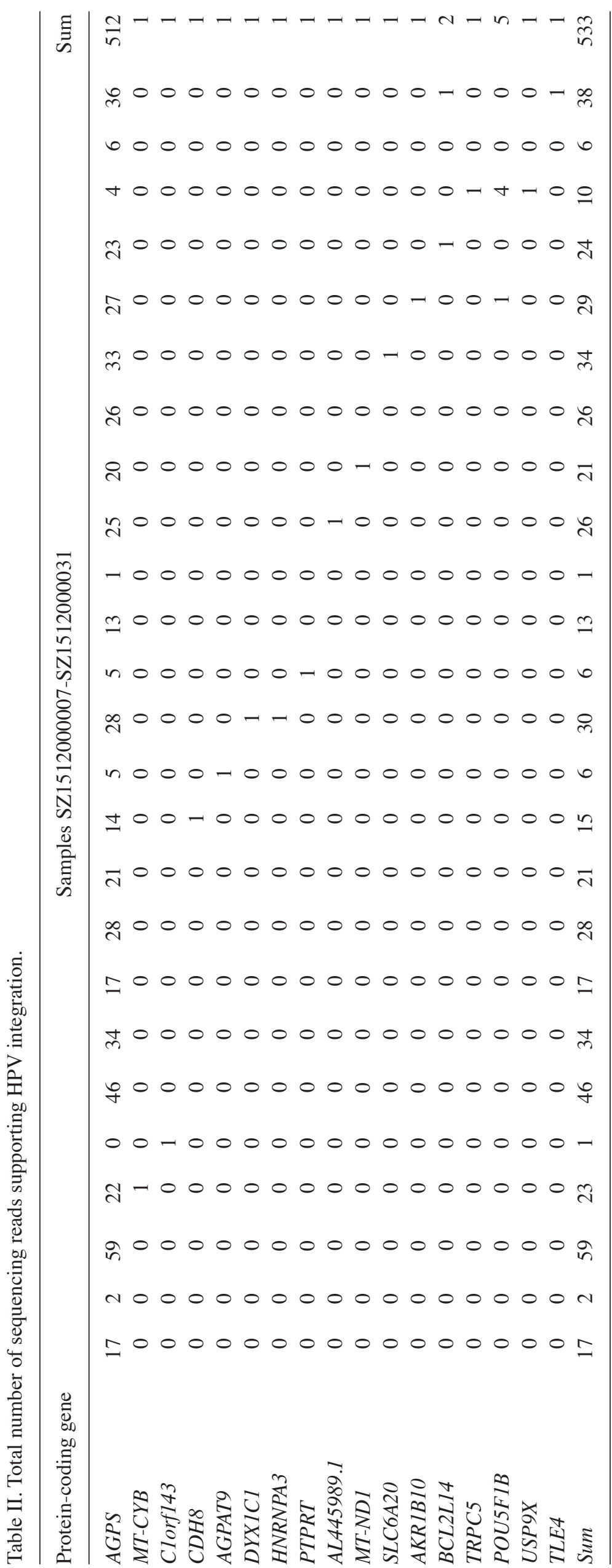


prior to or following radiotherapy tended to cluster together. Sequencing data revealed that the HPV type was HPV 16, which was consistent with the traditional detection method. HPV integration events were detected in POU5F1B in tumour cells following radiotherapy. The present study indicated that a proportion of tumour cells could survive radiotherapy, which informs on the mechanism of radiotherapy resistance.

Previous studies have suggested that radiotherapy resistance is the result of the intratumoural heterogeneity and subpopulation diversity of cancer cells $(34,35)$. The results of the present study demonstrated the subpopulation diversity of cancer cells in the cervical tumour prior to radiotherapy. Certain subpopulations may be sensitive to radiotherapy and could thus be effectively killed. If all subpopulations are sensitive to radiotherapy, the patient may recover following radiotherapy. However, if at least one subpopulation is resistant to radiotherapy, the radiotherapy may not be effective for the patient. The patient in the present study evidently belongs to the latter category.

Radiotherapy resistance could be predicted by expression analysis based on a defined set of genes (36). In addition to gene expression levels, somatic mutations are reported to be involved in radiotherapy resistance $(37,38)$. For example, all patients with medulloblastomas harbouring tumour protein p53 mutations experienced early recurrence, and the 5-year survival rate of these patients was $0 \%$ (37). Somatic mutations may also be involved in radiotherapy resistance in cervical cancer. For example, patients with KRAS proto-oncogene, GTPase-mutant cervical cancer who received radiation therapy experienced poorer outcomes (39). HPV integration is a specific type of somatic variant; thus, HP integration may also result in radiotherapy resistance. However, it has been reported that certain tumours with HPV integration may have enhanced radiation sensitivity, such as those of the head and neck (40), and squamous cell carcinomas (41). The HPV infection and the HPV type have also been shown to be associated with radiotherapy resistance (42).

However, to the best of our knowledge, no reported study has associated HPV integration sites or genes affected by HPV with radiotherapy resistance. In the present study, in tumour cells following radiotherapy, but not prior to radiotherapy, an HPV integration site in was detected in POU 5F1B, which may be a driver mutation in the tumour genome and thus responsible for the radiotherapy resistance. Several lines of evidence indicate that $P O U 5 F 1 B$ may be responsible for radiotherapy resistance: First, $P O U 5 F 1 B$ was reported to be a hotspot for HPV integration in cervical tumours $(17,32)$; second, amplifications of $P O U 5 F 1 B$ have been reported to promote an aggressive phenotype in gastric cancer (43); and third, POU $5 F 1 B$ is located at $8 \mathrm{q} 24$, a well-known susceptibility locus for various tumours $(44,45)$. These observations indicate that the HPV integration site in POU 5 F1B may responsible for radiotherapy resistance.

The current study presents an analysis of mutations prior to and following radiotherapy in a cervical tumour using single-cell sequencing. The results indicate that tumour cells with HPV integration in POU $5 F 1 B$ survive radiotherapy, and that tumour cells prior to and following radiotherapy exhibit distinct characteristics. The results obtained provide novel insights into the specific molecular events that drive radiotherapy resistance in cervical cancer.

\section{Acknowledgements}

The present study was supported by a grant from Beijing Municipal Science and Technology commission (no. D0906008040491).

\section{Competing interests}

The authors declare that they have no competing interests.

\section{References}

1. Parkin DM, Bray F, Ferlay J and Pisani P: Global cancer statistics, 2002. CA Cancer J Clin 55: 74-108, 2005.

2. Ferlay J, Shin HR, Bray F, Forman D, Mathers C and Parkin DM: Estimates of worldwide burden of cancer in 2008: GLOBOCAN 2008. Int J Cancer 127: 2893-2917, 2010.

3. DeSantis CE, Lin CC, Mariotto AB, Siegel RL, Stein KD, Kramer JL, Alteri R, Robbins AS and Jemal A: Cancer treatment and survivorship statistics, 2014. CA Cancer J Clin 64: 252-271, 2014.

4. Ravichandran R: Has the time come for doing away with Cobalt-60 teletherapy for cancer treatments. J Med Phys 34: 63-65, 2009.

5. Zhang YJ, Wei L, Li J, Zheng YQ and Li XR: Status quo and development trend of breast biopsy technology. Gland Surg 2: 15-24, 2013.

6. Malicki J: Medical physics in radiotherapy: The importance of preserving clinical responsibilities and expanding the profession's role in research, education, and quality control. Rep Pract Oncol Radiother 20: 161-169, 2015.

7. Tanderup K, Eifel PJ, Yashar CM, Potter R and Grigsby PW: Curative radiation therapy for locally advanced cervical cancer: Brachytherapy is NOT optional. Int J Radiat Oncol Biol Phys 88: 537-539, 2014.

8. McCormack M, Kadalayil L, Hackshaw A, Hall-Craggs MA, Symonds RP, Warwick V, Simonds H, Fernando I, Hammond M, James L, et al: A phase II study of weekly neoadjuvant chemotherapy followed by radical chemoradiation for locally advanced cervical cancer. Br J Cancer 108: 2464-2469, 2013.

9. Amichetti $\mathrm{M}$ and Amelio D: A review of the role of re-irradiation in recurrent high-grade glioma (HGG). Cancers (Basel) 3: 4061-4089, 2011 .

10. Hao JJ, Lin DC, Dinh HQ, Mayakonda A, Jiang YY, Chang C, Jiang Y, Lu CC, Shi ZZ, Xu X, et al: Spatial intratumoral heterogeneity and temporal clonal evolution in esophageal squamous cell carcinoma. Nat Genet 48: 1500-1507, 2016.

11. Yu C, Yu J, Yao X, Wu WK, Lu Y, Tang S, Li X, Bao L, Li X, Hou Y, et al: Discovery of biclonal origin and a novel oncogene SLC12A5 in colon cancer by single-cell sequencing. Cell Res 24: 701-712, 2014.

12. Pett M and Coleman N: Integration of high-risk human papillomavirus: A key event in cervical carcinogenesis? J Pathol 212: 356-367, 2007.

13. Chandrani P, Kulkarni V, Iyer P, Upadhyay P, Chaubal R, Das P, Mulherkar R, Singh R and Dutt A: NGS-based approach to determine the presence of HPV and their sites of integration in human cancer genome. Br J Cancer 112: 1958-1965, 2015.

14. Zhao R, Zhang WY, Wu MH, Zhang SW, Pan J, Zhu L, Zhang YP, $\mathrm{Li} \mathrm{H}, \mathrm{Gu}$ YS and Liu XZ: Human papillomavirus infection in Beijing, people's republic of China: A population-based study. $\mathrm{Br}$ J Cancer 101: 1635-1640, 2009.

15. Dai M, Bao YP, Li N, Clifford GM, Vaccarella S, Snijders PJ, Huang RD, Sun LX, Meijer CJ, Qiao YL and Franceschi S: Human papillomavirus infection in shanxi province, people's republic of China: A population-based study. Br J Cancer 95: 96-101, 2006

16. Li LK, Dai M, Clifford GM, Yao WQ, Arslan A, Li N, Shi JF, Snijders PJ, Meijer CJ, Qiao YL and Franceschi S: Human papillomavirus infection in shenyang city, people's republic of China: A population-based study. Br J Cancer 95: 1593-1597, 2006.

17. Hu Z, Zhu D, Wang W, Li W, Jia W, Zeng X, Ding W, Yu L, Wang X, Wang L, et al: Genome-wide profiling of HPV integration in cervical cancer identifies clustered genomic hot spots and a potential microhomology-mediated integration mechanism. Nat Genet 47: 158-163, 2015. 
18. Navin N, Kendall J, Troge J, Andrews P, Rodgers L, McIndoo J, Cook K, Stepansky A, Levy D, Esposito D, et al: Tumour evolution inferred by single-cell sequencing. Nature 472: 90-94, 2011.

19. Xu X, Hou Y, Yin X, Bao L, Tang A, Song L, Li F, Tsang S, $\mathrm{Wu} \mathrm{K}, \mathrm{Wu} \mathrm{H}$, et al: Single-cell exome sequencing reveals single-nucleotide mutation characteristics of a kidney tumor. Cell 148: 886-895, 2012.

20. Felthaus O, Ettl T, Gosau M, Driemel O, Brockhoff G, Reck A, Zeitler K, Hautmann M, Reichert TE, Schmalz G and Morsczeck C: Cancer stem cell-like cells from a single cell of oral squamous carcinoma cell lines. Biochem Biophys Res Commun 407: 28-33, 2011.

21. Koskas M and Rouzier R: Comparative performance of the 2009 International Federation of Gynecology and Obstetrics' staging system for uterine corpus cancer. Obstet Gynecol 117: 1225-1226; author reply 1226, 2011.

22. Sung WK, Zheng H, Li S, Chen R, Liu X, Li Y, Lee NP, Lee WH, Ariyaratne PN, Tennakoon C, et al: Genome-wide survey of recurrent $\mathrm{HBV}$ integration in hepatocellular carcinoma. Nat Genet 44: 765-769, 2012.

23. Kent WJ, Sugnet CW, Furey TS, Roskin KM, Pringle TH, Zahler AM and Haussler D: The human genome browser at UCSC. Genome Res 12: 996-1006, 2002.

24. Li H and Durbin R: Fast and accurate short read alignment with Burrows-Wheeler transform. Bioinformatics 25: 1754-1760, 2009.

25. Li H: A statistical framework for SNP calling, mutation discovery, association mapping and population genetical parameter estimation from sequencing data. Bioinformatics 27 2987-2993, 2011.

26. Koboldt DC, Zhang Q, Larson DE, Shen D, McLellan MD, Lin L, Miller CA, Mardis ER, Ding L and Wilson RK: VarScan 2: Somatic mutation and copy number alteration discovery in cancer by exome sequencing. Genome Res 22: 568-576, 2012.

27. 1000 Genomes Project Consortium, Auton A, Brooks LD, Durbin RM, Garrison EP, Kang HM, Korbel JO, Marchini JL, McCarthy S, McVean GA and Abecasis GR: A global reference for human genetic variation. Nature 526: 68-74, 2015.

28. Cingolani P, Platts A, Wang le L, Coon M, Nguyen T, Wang L, Land SJ, Lu X and Ruden DM: A program for annotating and predicting the effects of single nucleotide polymorphisms, SnpEff: SNPs in the genome of Drosophila melanogaster strain w1118; iso-2; iso-3. Fly (Austin) 6: 80-92, 2012.

29. Kuong KJ and Loeb LA: APOBEC3B mutagenesis in cancer. Nat Genet 45: 964-965, 2013.

30. Ojesina AI, Lichtenstein L, Freeman SS, Pedamallu CS, Imaz-Rosshandler I, Pugh TJ, Cherniack AD, Ambrogio L, Cibulskis K, Bertelsen B, et al: Landscape of genomic alterations in cervical carcinomas. Nature 506: 371-375, 2014.

31. Sabatier L, Lebeau J and Dutrillaux B: Radiation-induced carcinogenesis: Individual sensitivity and genomic instability. Radiat Environ Biophys 34: 229-232, 1995.

32. Zhang R, Shen C, Zhao L, Wang J, McCrae M, Chen X and Lu F: Dysregulation of host cellular genes targeted by human papillomavirus (HPV) integration contributes to HPV-related cervical carcinogenesis. Int J Cancer 138: 1163-1174, 2016.
33. Annunziata C, Buonaguro L, Buonaguro FM and Tornesello ML: Characterization of the human papillomavirus (HPV) integration sites into genital cancers. Pathol Oncol Res 18: 803-808, 2012.

34. Marusyk A and Polyak K: Tumor heterogeneity: Causes and consequences. Biochim Biophys Acta 1805: 105-117, 2010.

35. Xia Q, Guo Y, Zhang Z, Li D, Xuan Z, Li Z, Dai F, Li Y, Cheng D, Li R, et al: Complete resequencing of 40 genomes reveals domestication events and genes in silkworm (Bombyx). Science 326: 433-436, 2009

36. Eschrich S, Zhang H, Zhao H, Boulware D, Lee JH, Bloom G and Torres-Roca JF: Systems biology modeling of the radiation sensitivity network: A biomarker discovery platform. Int J Radiat Oncol Biol Phys 75: 497-505, 2009.

37. Tabori U, Baskin B, Shago M, Alon N, Taylor MD, Ray PN, Bouffet E, Malkin D and Hawkins C: Universal poor survival in children with medulloblastoma harboring somatic TP53 mutations J Clin Oncol 28: 1345-1350, 2010.

38. Krakstad C and Chekenya M: Survival signalling and apoptosis resistance in glioblastomas: Opportunities for targeted therapeutics. Mol Cancer 9: 135, 2010

39. Sreelekha TT, Nair MK, Jayaprakash PG and Pillai MR: Immunophenotype of mutant ras p21 and early response to radiotherapy in cancer of the uterine cervix. J Exp Clin Cancer Res 18: 337-341, 1999.

40. Kimple RJ, Smith MA, Blitzer GC, Torres AD, Martin JA, Yang RZ, Peet CR, Lorenz LD, Nickel KP, Klingelhutz AJ, et al: Enhanced radiation sensitivity in HPV-positive head and neck cancer. Cancer Res 73: 4791-4800, 2013.

41. Vozenin MC, Lord HK, Hartl D and Deutsch E: Unravelling the biology of human papillomavirus (HPV) related tumours to enhance their radiosensitivity. Cancer Treat Rev 36: 629-636, 2010.

42. Badaracco G, Savarese A, Micheli A, Rizzo C, Paolini F, Carosi M, Cutillo G, Vizza E, Arcangeli G and Venuti A: Persistence of HPV after radio-chemotherapy in locally advanced cervical cancer. Oncol Rep 23: 1093-1099, 2010.

43. Hayashi H, Arao T, Togashi Y, Kato H, Fujita Y, De Velasco MA, Kimura H, Matsumoto K, Tanaka K, Okamoto I, et al: The OCT4 pseudogene POU5F1B is amplified and promotes an aggressive phenotype in gastric cancer. Oncogene 34: 199-208, 2015.

44. Pomerantz MM, Ahmadiyeh N, Jia L, Herman P, Verzi MP, Doddapaneni H, Beckwith CA, Chan JA, Hills A, Davis M, et al: The 8q24 cancer risk variant rs6983267 shows long-range interaction with MYC in colorectal cancer. Nat Genet 41: 882-884, 2009.

45. Yeager M, Orr N, Hayes RB, Jacobs KB, Kraft P, Wacholder S, Minichiello MJ, Fearnhead P, Yu K, Chatterjee N, et al: Genome-wide association study of prostate cancer identifies a second risk locus at 8q24. Nat Genet 39: 645-649, 2007.

This work is licensed under a Creative Commons Attribution-NonCommercial-NoDerivatives 4.0 International (CC BY-NC-ND 4.0) License. 